\title{
Optimisation des réseaux de distribution des produits agroalimentaires : Modélisation et application
}

\author{
Salima Kendi*, Mohammed Said Radjef**, Abdelhakim Hammoudi***
}

\author{
DOI: $10.30682 / \mathrm{nm} 2004 \mathrm{~d}$ \\ JEL codes: B23, C02, C61
}

\begin{abstract}
A certain number of factors can affect the functioning of the distribution chains of large agrifood companies and impact their efficiency. Among them, poor adaptation to fluctuations in demand, imperfect control of production and transport costs and poor location of distribution centers. These dysfunctions can have negative consequences with high product prices, supply disruptions and customer dissatisfaction. The company is therefore required to review regularly the distribution plan for its products in order to optimize it: addition, deletion, relocation, reassignment of warehouses, delivery centers, etc. We propose, through a case study of the agro-food group Cevital (Algeria), a solution to the problem of restructuring supply and distribution networks, at a stage of development of this company. Using an operational research tool (mixed-integer linear programming), the study assesses four scenarios for structuring the company's distribution network and suggests, for decision-making purposes, strategic solutions for managers.
\end{abstract}

Keywords: Agri-food industry, Supply-chain, Management, Modeling, Location, Distribution centers, Transport cost, Cevital group, Mediterranean region.

\section{Introduction}

L'alimentation fut longtemps un fait essentiellement agricole. Elle est assurée aujourd'hui à travers un processus multisectoriel de production, transformation et de distribution faisant appel à un grand nombre d'activités différentes et d'agents économiques de plus en plus nombreux. Ces agents interagissent dans le cadre de ce qui est communément appelé supply chain. Leur diversité et le développement des activités en amont et en aval de l'agriculture conduisent à des réseaux complexes avec de nouvelles préoccupations en termes de circulation des produits alimentaires depuis leur production jusqu'à leur consommation (Padilla et Bencharif, 2001). La croissance de la demande en produits agroalimentaires, du fait notamment de l'augmentation de la population, accentue les défis d'organisation des supply chains.

L'offre alimentaire est de plus en plus assurée à travers un réseau complexe regroupant un ensemble d'activités en interaction, incluant la

\footnotetext{
* Unité de Recherche LaMOS (Modélisation et Optimisation des Systèmes), Faculté des Sciences de la Nature et de la Vie, Université de Bejaia, Bejaia, Algérie.

** Unité de Recherche LaMOS (Modélisation et Optimisation des Systèmes), Département de Recherche Opérationnelle, Faculté des Sciences Exactes, Université de Bejaia, Bejaia, Algérie.

*** Université Paris-Saclay, INRAE, UR ALISS, Ivry-sur-Seine, France.

Corresponding author: salima_kendi@yahoo.fr
} 
production/transformation, la collecte, le stockage, le conditionnement, la commercialisation (marchés de gros ou de détail, centres de distribution). Les questions liées à la distribution, à l'entreposage, au transport de marchandises, la satisfaction juste à temps et sans rupture de la clientèle, sont une préoccupation constante, en particulier pour les grandes entreprises du secteur, que ces activités soient d'ailleurs intégrées ou assurées par des partenaires extérieurs. En effet, un certain nombre de facteurs peut affecter le fonctionnement d'une chaîne d'approvisionnement et de distribution, réduisant la valeur créée en son sein, notamment les aléas de la demande, la non maitrise des coûts de production et des coûts de transport, des localisations inadéquates des entrepôts, des centres de distribution, etc. L'entreprise doit subir de telles inefficacités des fonctions logistiques quand celles-ci sont externalisées. Elles ont une plus grande flexibilité stratégique pour en améliorer le fonctionnement quand ces fonctions sont intégrées en leur sein de l'entreprise. Les entreprises qui disposent de leur propre réseau logistique doivent alors anticiper au mieux les fluctuations de marché pour décider de façon optimale les caractéristiques de leur réseau. Ils doivent, le cas échéant, ajuster quand cela est nécessaire leur infrastructure à des stades postérieurs de leur développement (ajout, suppression, relocalisation et/ou réaffectation d'entrepôts, de centres de livraison...).

Il est fréquent en effet qu'à un stade de son développement, l'entreprise soit contrainte de redéfinir le dimensionnement de son réseau et/ ou de décider de son extension en tenant compte à la fois de son expérience passée et des nouvelles données du marché. Devant la difficulté de prédire précisément l'évolution des marchés, certains choix de localisation et des plans de distribution, qui pouvaient apparaître efficaces à une étape du développement de l'entreprise, peuvent s'avérer inappropriés à une autre. Les ajustements passent alors le plus souvent par la remise en cause des choix antérieurs, souvent les plus lourds en termes d'investissement.

L'objectif de cet article est justement de proposer, à travers un modèle de localisation et de relocalisation des infrastructures logistiques, des solutions pour rationaliser les décisions du groupe agro-alimentaire algérien Cevital. Nous allons montrer tout d'abord à travers cette étude de cas, comment cet outil de recherche opérationnelle, au-delà des aspects mathématiques nécessaires à la résolution de la question posée par l'entreprise, peut s'avérer particulièrement utile à éclairer la décision privée. L'analyse et la résolution du problème d'optimisation conclut à une nécessité de redimensionnement du réseau de l'entreprise (localisation, ajout ou suppression de certaines infrastructures), afin d'améliorer au mieux les performances de cette dernière. Le modèle, construit à partir des questions concrètes que se pose une entreprise a toutefois une valeur de généralité. Il peut être adapté aux spécificités d'autres entreprises en prenant en compte leurs contraintes et environnement propres.

\section{Etat de lieu et littérature économique}

Historiquement, les fondements de la théorie économique de la localisation appliquée à l'agriculture ont été posés par Von Thünen (1826) dans son ouvrage intitulé Isolated State with Respect to Agriculture and National Economy. L'idée est d'expliquer la localisation des activités agricoles, l'utilisation des sols et la formation de la rente en mettant en évidence le rôle de la distance entre les bassins de production et les places de marché (et in fine les coûts de transports qui y sont associés). Plus tard, s'est développé un certain nombre de théories économiques de la localisation, qui diffèrent essentiellement par la nature des questions spécifiques étudiées et le corpus d'hypothèses pris en compte : environnement des acteurs (typologies des marchés, structure des systèmes de production et de la demande, des coûts de transport...), comportement des acteurs (faiseurs ou preneurs de prix, nature des variables de décision, typologie des jeux d'interaction sur les marchés...). Reprenant une classification proposée par Ponsard et Beguin (1988), on peut citer entre autres, les travaux de Weber (1909) avec sa théorie de la localisation industrielle et la détermination du coût minimum de transport, le modèle d'Hotelling (1929) analysant, dans le cadre de la nouvelle économie industrielle, les liens entre la localisation des entreprises et la concurrence sur le marché (prix 
et parts de marché). La théorie, communément appelée " nouvelle économie géographique » (Krugman, 1998), se pose comme objectif d'expliquer les mécanismes qui déterminent la localisation des systèmes de production et des activités agro-industrielles (forces d'agglomération et de dispersion (Fujita et Thisse, 1997).

Une branche récente de la littérature sur la question de la localisation se concentre sur la réalité des échanges et des flux commerciaux au sein des chaînes d'approvisionnement et des filières. Il s'agit d'analyser, par exemple, les effets de la localisation des marchés de gros (Etemadnia et al., 2015 ; Zheng et al., 2019 ; Biuki et al., 2020) ou des bassins de production (Sarkar et al., 1997 ; Rhim et al., 2003) sur le fonctionnement des marchés et la formation des prix. Parallèlement à cette littérature d'essence économique, une autre approche, fondée sur les outils de recherche opérationnelle, sans formalisation des questions de comportement et de marché, s'est développée autour de préoccupations plus concrètes : la recherche de solutions techniques pour améliorer la gestion et le management des opérations le long des chaînes d'approvisionnement et de distribution (ReVelle et Eiselt, 2005 ; Baumol et Wolfe, 1976). Un large panel de questions concrètes s'est dès lors imposé : Quels délais d'acheminement des produits? Quels coûts et pour quels prix ? Quel nombre, quelle taille et quelle localisation des centres de production et de distribution? Quelle technologie utiliser dans la production/transformation ? Quelle serait la modalité optimale de transport, de stockage? Quels sont les produits à fabriquer et à stocker dans chaque entrepôt ?

A partir de modèles mathématiques, les travaux, plutôt théoriques, proposent des solutions techniques pour optimiser le fonctionnement des réseaux logistiques en déterminant les caractéristiques souhaitables (taille des centres de distribution ; des entrepôts, leurs localisations...). Ces travaux portent sur différentes typologies de réseaux, intégrant différentes contraintes de l'environnement de la chaîne : zones géographiques éligibles, localisation, comportements des acteurs, nature des flux de produits, structure de la demande, fonction objectif du décideur ${ }^{1}$. Au niveau méthodologique, il est utile de préciser ce qui distingue les modèles de localisation et les modèles de distribution (optimisation des chaines de distribution). Un modèle de distribution est une extension d'un modèle de localisation discret auquel sont ajoutées certaines variables et contraintes, notamment de flux de marchandises et de coûts de transport. Le modèle de localisation analyse la localisation des entrepôts en fonction de celles des usines et des clients (Balinski, 1965 ; Klose et Drexl, 2005 ; Melo et al., 2009). Il permet, comme décrit dans Francis et al. (1983), de localiser des établissements tenant compte de certains coûts, comme ceux de transport et de construction. Le modèle de Geoffrion et Graves (1974) a été le premier modèle de distribution à intégrer la localisation des centres de distribution et l'affectation des produits et des clients à ces derniers afin de minimiser le coût total du système. Dans la lignée de cette littérature, Ahmadi-Javid et Hoseinpour (2015) proposent un programme non linéaire à variables mixtes, pour déterminer les localisations optimales des centres de distribution à approvisionner. Le modèle prend en compte la variation de la demande en fonction des prix et des contraintes de capacités de stockage. Le modèle, à la différence de Cortinhal et al. (2015), ne traite cependant pas la question de redimensionnement d'un réseau logistique. Dans le prolongement de cette approche, Tang et al. (2016) considèrent un réseau collaboratif (entre un cluster de fournisseurs et plusieurs milliers de clients) et Ma et al. (2016) un modèle de localisation (formellement, une programmation bi-niveaux) pour la gestion de la chaîne d'approvisionnement.

Le modèle de localisation-allocation que nous proposons est dans la lignée de cette littérature et se pose comme objectif l'optimisation et le

\footnotetext{
${ }^{1}$ Parmi les questions traitées : nombre et localisation des installations pour minimiser les coûts de transport et/ou satisfaire la demande, schéma de circulation des flux pour garantir la qualité des produits et la traçabilité, nombre et emplacement des hubs alimentaires, allocation de zones géographiques aux clients...
} 
redimensionnement du réseau de distribution de Cevital. Nous analysons différents scénarios associés à la structure du réseau de cette entreprise. Sur un plan technique, il renvoie à un programme linéaire mixte (MILP) ${ }^{2}$.

\section{Position du problème et modélisation}

En Algérie, le secteur des industries agroalimentaires a connu une progression vigoureuse au cours de ces 20 dernières années. Les perspectives de croissance à venir sont a priori prometteuses au regard de l'importance de la demande et des possibilités d'exportation dans certains secteurs (Bennacer, 2018). Le secteur privé à plus de $80 \%$ occupe une place de choix dans l'approvisionnement du marché national en produits qui constituent la base du système alimentaire et nutritionnel algérien (farine, semoule, pâtes alimentaires, lait et produits dérivés, huiles alimentaires, tomate industrielle, sucre, etc.) (Bessaoud et al., 2019).

Le groupe industriel Cevital représente l'une des figures clé de ce paysage. Le Groupe est un conglomérat algérien de l'industrie agroalimentaire, la grande distribution, l'industrie et les services. Créé en 1998 sur des fonds privés (Société Par Actions), le groupe est parmi les entreprises algériennes qui ont vu le jour dès l'entrée de l'Algérie en économie de marché, pour constituer aujourd'hui un acteur incontournable dans l'économie nationale, en raison de sa taille, sa diversification et son déploiement à l'échelle nationale. Le groupe occupe également les premières places en matière de création d'emploi et de distribution de la richesse et, par conséquent, apporte une forte contribution au budget de l'Etat. Il compte, par ailleurs, parmi ses clients de grands noms du domaine de l'agro-business, citons : Coca-Cola, Kraft Food, Danone, etc. (ANPCE, 2019).

Composé de plusieurs unités de production (les raffineries de sucre et des huiles, la margarinerie, le conditionnement d'eau minérale ainsi que la fabrication et le conditionnement de boissons rafraichissantes et conserverie), le secteur agro-alimentaire représente la pierre angulaire du groupe Cevital $(80 \%$ de ses activités $\left.{ }^{3}\right)$ et lui procure une très bonne position sur le marché national (parmi un certain nombre de producteurs nationaux et importateurs) et en Afrique. L'implantation de l'usine de production des huiles, de sucre et de margarine au sein du port de Béjaia a permis à Cevital de disposer de silos portuaires et de l'accès direct à un quai du port pour le déchargement des matières premières et l'exportation de ses produits. Grace à ses filières, le groupe Cevital devient le plus grand groupe privé en Algérie et le deuxième exportateur après l'entreprise pétrolière SONATRACH. Une grande partie de sa production est exportée dans plusieurs pays européens (France, Espagne, Italie, Allemagne) et d'autres pays de l'Europe orientale, d'Afrique, du Moyen Orient et du Maghreb.

Pour assurer une bonne couverture de ses produits en les rapprochant le mieux possible des clients dans les délais les plus courts et au meilleur prix, le groupe Cevital a développé son propre réseau de distribution avec la création de ses filiales Numilog (2012) et Numidis (2007). Ainsi, le transport journalier de la marchandise depuis les trois unités de production jusqu'aux trois plateformes et des plateformes aux CLRs est assuré par le groupe Cevital. Les coûts de transport des produits depuis les CLRs ou depuis les unités de production aux clients sont à la charge des clients.

Le développement continu du groupe $\mathrm{Ce}$ vital et l'évolution de son environnement socio-économique imposent à son réseau de distribution une mise à niveau régulière de ses moyens logistiques et de transport et une actualisation de ses plans de distribution pour maintenir sa performance.

Pour répondre à ces questions, nous construisons dans une première étape un modèle général d'optimisation d'un réseau de distribution de produits pour une entreprise agroalimentaire avant de l'appliquer, dans la section

\footnotetext{
${ }^{2}$ La résolution du modèle s'effectue en utilisant la version CPLEX Community 12.6.3 comme solveur du MILP.

3 https://www.cevital.dz/agro-industrie-et-distribution/.
} 
Figure 1 - Système type d'une chaine logistique de distribution de produits pour les entreprises agroalimentaires.

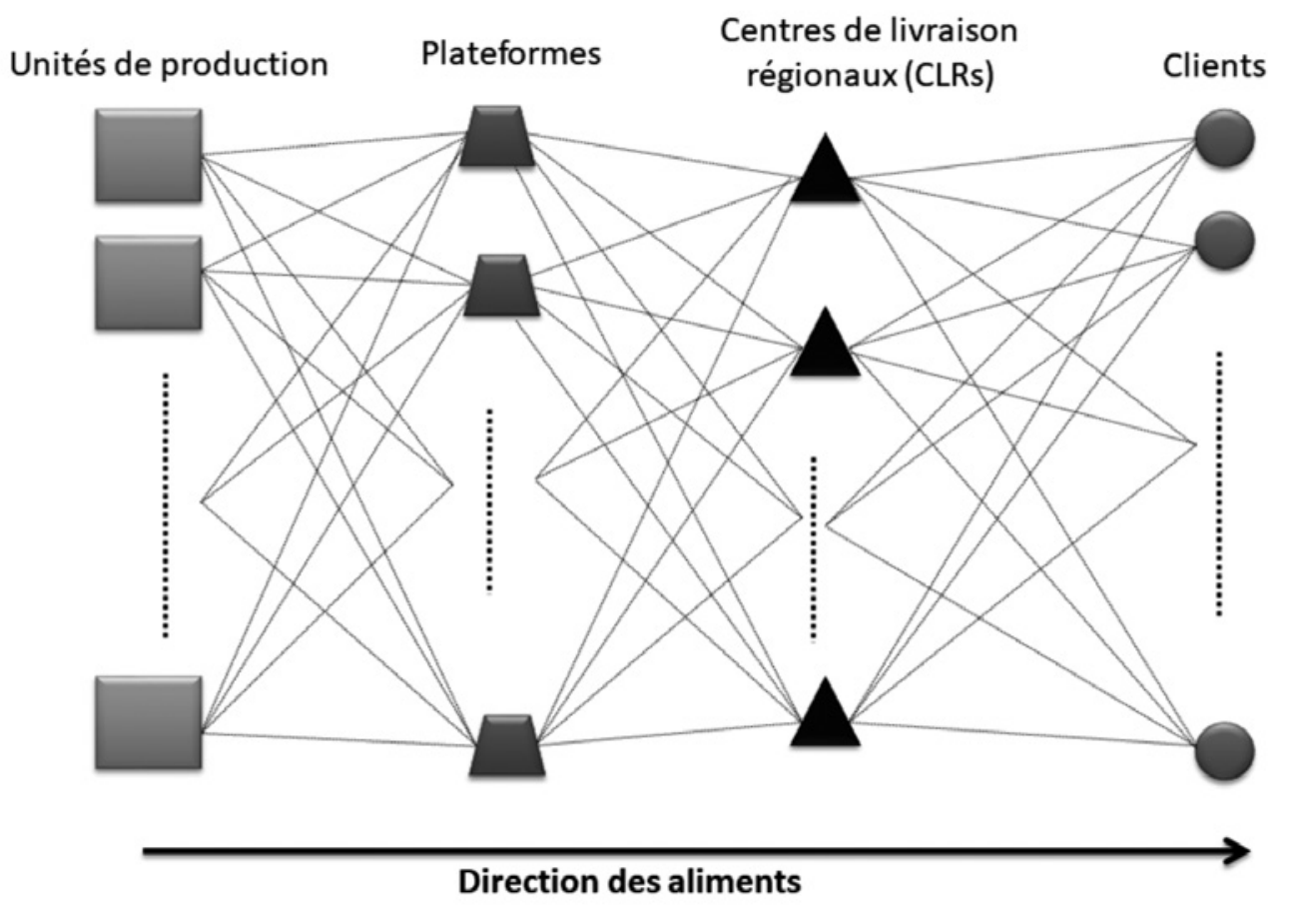

Source: réalisée par les auteurs.

suivante, aux données factuelles attachées à une problématique spécifique posée par Cevital.

Un système typique d'une chaine logistique de distribution de produits pour les entreprises agroalimentaires est illustré dans la Figure 1, où les nœuds représentent des unités de production, des plateformes (ou des entrepôts) et des centres de livraison régionaux (CLRs). Les arêtes de liaison représentent les flux de marchandises entre les nœuds.

Pour redimensionner d'une manière optimale et mettre à jour cette infrastructure existante, nous sommes amenés à répondre aux questions suivantes :

- Que se passera-t-il si nous ajoutons des plateformes et/ou des CLRs ? Où les localiser? A quelles tailles et à quel nombre ?

- Que se passera-t-il si nous fermons des plateformes et/ou des CLRs? Lesquels?

- Quelles réaffectations (optimales) entre les unités de production, les plateformes, les CLR et les clients?
Les réponses à ces questions vont constituer des décisions critiques, permettant de minimiser le coût total du réseau de distribution, qui inclut :

- le coût total de transport de l'entreprise ;

- le coût total de transport des clients ;

- le coût total de maintien des plateformes et des CLRs ;

- le coût total de fermeture des plateformes et des CLRs ;

- le coût total d'installation de nouvelles plateformes et CLRs.

En notant par :

- $x$ : le vecteur des décisions à prendre, dont les composantes désigneront : les quantités de produit à transporter d'une destination à une autre du réseau, les plateformes en activité à fermer et les nouvelles à construire, les CLR à ouvrir et ceux à fermer ;

- $X$ : l'ensemble des solutions réalisables vérifiant les contraintes relatives au réseau de distribution (capacités, satisfaction de la demande...); 
- $\varphi$ la fonction du coût total du réseau de distribution agrégeant les coûts de transport de l'entreprise et ceux des clients; le coût de maintien des plateformes et des CLRs en activités, les coûts de fermeture des plateformes et des CLRs en activité et les coûts de construction (fixe et variable) de nouvelles plateformes et de nouveaux CLRs.

Le problème d'optimisation d'un réseau de distribution d'une entreprise agroalimentaire se ramène à la résolution du programme linéaire mixte: (voir annexe 2 pour la description plus détaillée du modèle) :

$$
\min _{x \in X} \varphi(x)
$$

\section{4. Étude de cas: optimisation du réseau de distribution du groupe Cevital}

Face au développement du groupe Cevital, de nouveaux besoins se sont fait sentir pour améliorer les performances de son réseau de distribution. Les fréquentes ruptures de stocks constatées au sein de certains CLRs du centre du pays en raison de la progression de la demande dans cette région et pour se rapprocher des clients du Sud, qui s'approvisionnaient depuis les trois unités de production du Nord, le groupe Cevital a pris la décision stratégique de localiser deux nouvelles plateformes sur deux régions : l'une à Alger, au Nord du pays, et l'autre à Biskra, au Sud. Il projette aussi de délocaliser certains de ses CLRs qui ne sont pas rentables, car ils génèrent des coûts de stockage avec de faibles taux d'occupation. Dans le but d'acheminer les produits des unités de production aux clients à moindre coût, les gestionnaires de Cevital nous ont soumis la problématique suivante : Quelles seraient les tailles optimales des plateformes d'Alger et de Biskra, si ces dernières venaient éventuellement à être installées ? Quelles seront les affectations optimales des CLRs à la plateforme d'Alger ?
Quelles seront les affectations optimales des clients à la plateforme de Biskra ? Et enfin, quels seront les CLRs à supprimer?

Nous développons une application du modèle (1) pour l'optimisation et le redimensionnement du réseau de distribution de Cevital, qui répond à cette problématique.

Le réseau initial de distribution du groupe $\mathrm{Ce}$ vital se compose $\mathrm{de}^{4}$ :

a) 3 unités de production spécialisées localisées comme suit :

- Bejaia usine, située au port de Béjaia : unité de production et de conditionnement de sucre, de margarine et de différentes catégories d'huile ;

- Cojek usine, située à El Kseur (Wilaya de Béjaia) : unité de production des boissons fruitées et des conserves ;

- LLK usine, située à Tizi-Ouzou : unité de production et de conditionnement des eaux minérales (plates, gazeuses et sodas).

b) 3 plateformes de stockage externes qui sont propres à l'entreprise : Bouira au Centre, Oran (Hassi-Ameur) à l'Ouest et Constantine (El-Khroub) à l'Est.

c) 18 Centres de Livraison Régionaux $(\mathrm{CLRs})^{5}$ répartis comme suit :

- 7 CLRs reliés à la plateforme d'Oran, qui sont : Sidi-Bel-Abbas (22), Oran (31), Relizane (48), Mostaganem (27), Tlemcen (13), Tiaret (14) et Mascara (29) ;

- 6 CLRs reliés à la plateforme de Bouira, qui sont : Alger (16), Blida (09), Boumerdès (35), Tizi-Ouzou (15), Bejaia (06) et Médéa (26) ;

- 5 CLRs reliés à la plateforme de Constantine, qui sont : Batna (05), Constantine (25), Sétif (19), Oum El Bouaghi (04) et Annaba (23).

d) de grands clients, dont les grossistes, les représentants, les industriels, qui sont dispersés sur tout le territoire national. Il s'agit de :

\footnotetext{
${ }^{4}$ Les plateformes, les CLRs et les clients prennent le nom et le code administratif de leur wilaya.

5 Etablissement créé par l'entreprise en 2014 pour réduire la pression sur le complexe et rapprocher la marchandise du client. Il est doté d'un centre d'appels pour gérer les demandes et possède une base de données de tous les clients.
} 
Figure 2 - Le réseau de production-distribution de l'entreprise Cevital sur le territoire national.

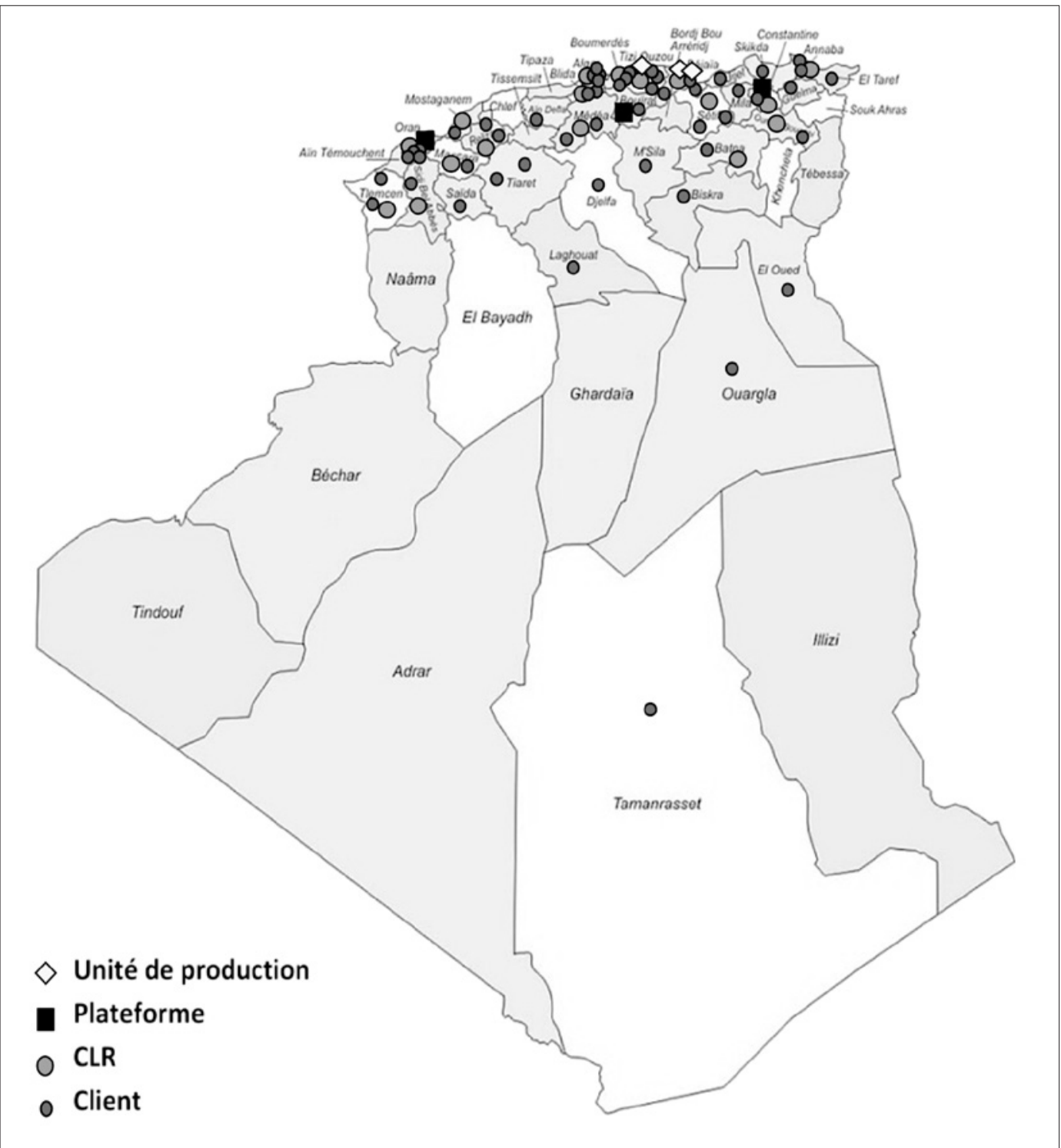

Source: réalisée par les auteurs à partir de la carte géographique de l'Algérie.

- 26 clients qui s'approvisionnent uniquement des CLRs (clients-CLR);

- 26 clients qui s'approvisionnent uniquement des unités de production (clients hors-CLRs).

Par ailleurs, nous illustrons sur la carte géographique de l'Algérie (Figure 2), le réseau de production-distribution de l'entreprise Cevital sur le territoire national.
Le plan de transport de marchandise adopté par Cevital est comme suit :

- le transport de la marchandise depuis les 3 unités de production jusqu'aux 3 plateformes et des plateformes aux CLRs est assuré par Cevital ;

- le coût de transport des produits depuis les CLRs ou les unités de production jusqu'aux clients est à la charge des clients eux-mêmes, 
Figure 3 - Le nouveau réseau envisagé par l'entreprise Cevital.

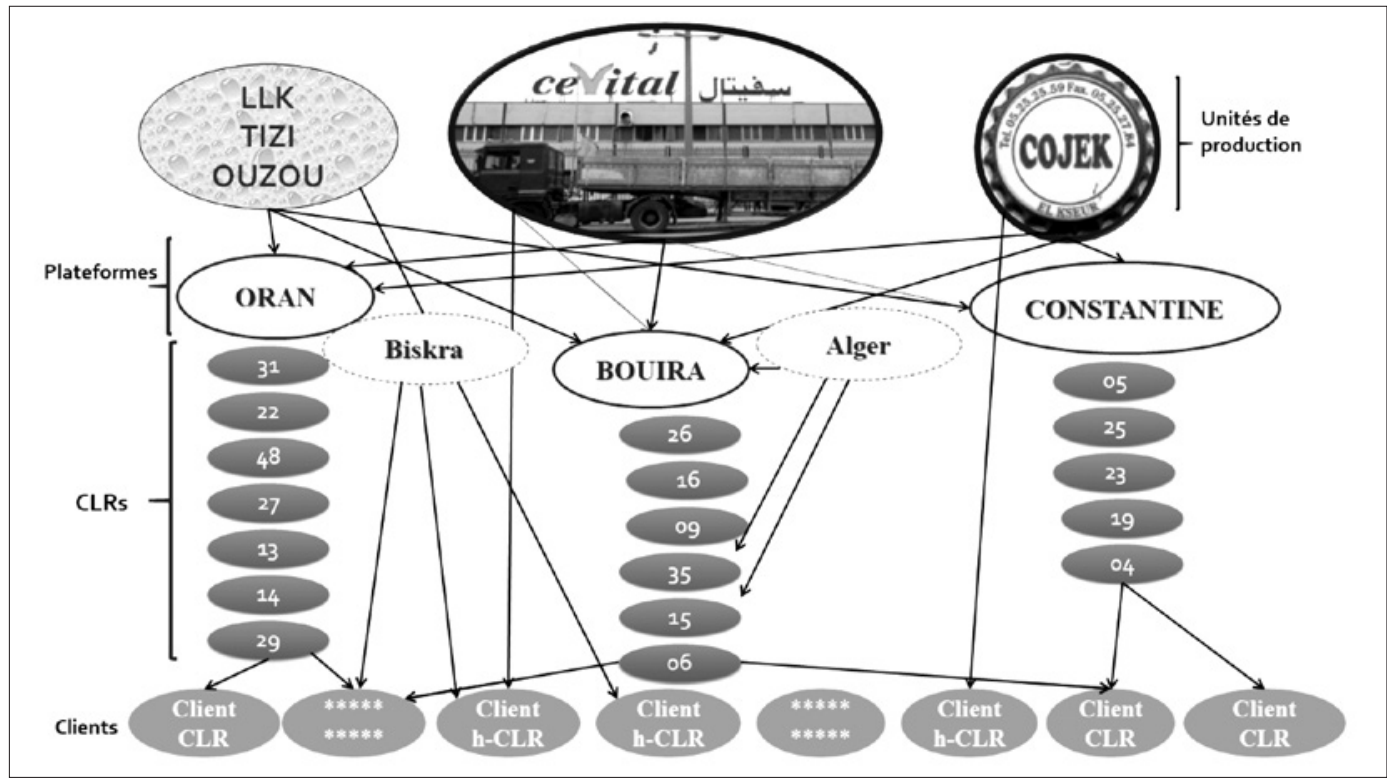

sachant que les clients-CLR s'approvisionnent des CLRs et les clients hors-CLR s'approvisionnent des 3 unités de production.

La problématique de Cevital consiste alors, d'une part, à déterminer les tailles des plateformes d'Alger et/ou de Biskra et, d'autre part, à identifier les CLRs qui s'approvisionneront à partir de la plateforme d'Alger, à déterminer les clients qui s'approvisionneront de la plateforme de Biskra et à décider des éventuels CLRs à supprimer. Enfin, notre travail consistera à définir la configuration optimale du réseau global de distribution de Cevital.

Sur la Figure 3, nous schématisons le nouveau réseau envisagé par l'entreprise.

\subsection{Collecte de données}

La collecte de données de l'année 2018 a été effectuée au niveau des différents services de l'entreprise Cevital. Au niveau du service production, nous avons collecté des données sur la capacité de production et fait un inventaire de sa gamme de produits (Cf. Tableau 1).

Dans le Tableau 2, nous représentons la liste des plateformes de l'entreprise Cevital avec leurs capacités de stockage, que nous avons recueillie au niveau du service commercial.
Tableau 1 - Capacité de production journalière de chaque produit.

\begin{tabular}{|c|c|c|}
\hline $\begin{array}{c}N^{\circ} d u \\
\text { produit }\end{array}$ & Produit & $\begin{array}{c}\text { Capacité de } \\
\text { production } \\
\text { (palette/jour) }\end{array}$ \\
\hline 1 & Huile & 14203 \\
\hline 2 & Sucre & 9024 \\
\hline 3 & Margarine & 3426 \\
\hline 4 & Eau minérale & 6495 \\
\hline 5 & $\begin{array}{c}\text { Boissons fruitées et } \\
\text { conserves }\end{array}$ & 1299 \\
\hline
\end{tabular}

Source : Réalisé par les auteurs, à partir des données collectées au niveau du groupe Cevital.

Tableau 2 - Les capacités des plateformes.

\begin{tabular}{|c|c|c|}
\hline Localisation & Plateformes & $\begin{array}{c}\text { Capacité } \\
\text { (palettes) }\end{array}$ \\
\hline Centre & Bouira & 20000 \\
\hline Est & $\begin{array}{c}\text { Constantine } \\
\text { (El Khroub) }\end{array}$ & 3000 \\
\hline Ouest & $\begin{array}{c}\text { Oran } \\
\text { (Hassi-Ameur) }\end{array}$ & 12000 \\
\hline
\end{tabular}

Source : Réalisé par les auteurs, à partir des données collectées au niveau du groupe Cevital. 
Tableau 3 - Coût de stockage d'une palette dans les CLRs.

\begin{tabular}{|c|c|c|c|}
\hline CLR & $\begin{array}{c}\text { Coût (DA) (palettel } \\
\text { jour) }\end{array}$ & CLR & $\begin{array}{c}\text { Coût (DA) (palettel } \\
\text { jour) }\end{array}$ \\
\hline Oum El Bouaghi (04) & 798 & Sidi Bel Abbas (22) & 1440 \\
\hline Batna (05) & 739 & Annaba (23) & 570 \\
\hline Bejaia (06) & 1856 & Constantine (25) & 1348 \\
\hline Blida (09) & 1014 & Médéa (26) & 1148 \\
\hline Tlemcen (13) & 713 & Mostaganem (27) & 939 \\
\hline Tiaret (14) & 723 & Mascara (29) & 713 \\
\hline Tizi-Ouzou (15) & 595 & Oran (31) & 482 \\
\hline Alger (16) & 775 & Boumerdès (35) & 879 \\
\hline Sétif (19) & 714 & Relizane (48) & 718 \\
\hline
\end{tabular}

Source : Réalisé par les auteurs, à partir des données collectées au niveau de Cevital.

Au niveau du service logistique, nous avons collecté les données suivantes :

- le coût de transport : $89 \mathrm{DA} / \mathrm{km}$;

- le coût moyen de stockage journalier (DA/ palette) dans chaque CLR (représentant la somme des coûts de stockage, d'allocation et de manutention) est présenté dans le Tableau 3.

Les données concernant les demandes ${ }^{6}$ des clients CLRs pour l'année 2018 sont représentées dans le Tableau 4 (voir annexe 1). Pour ne pas surcharger le papier, nous avons omis de présenter les demandes des clients hors-CLR.

Par ailleurs, nous avons utilisé l'application Web « Google Map » pour calculer certaines des distances inter-wilayas que nous avons utilisées dans le modèle.

\subsection{Application du modèle et étude de scénarios}

L'application du modèle mathématique (1) à la problématique spécifique de Cevital nous conduit à l'étude de quatre scénarios :
- Scénario 1 : modélise le réseau actuel de l'entreprise (sans la mise en activité des plateformes d'Alger ${ }^{7}$ et de Biskra) ;

- Scénario 2 : modélise le réseau avec la mise en activité de la plateforme d'Alger ;

- Scénario 3 : modélise le réseau avec la mise en activité de la plateforme de Biskra ;

- Scénario 4 : modélise le réseau envisagé par Cevital (avec la mise en activité des deux plateformes d'Alger et de Biskra).

Pour chacun des quatre scénarios, l'entreprise cherche, d'une part, à délocaliser d'une manière optimale les CLRs en surplus afin de réduire les coûts et, d'autre part, à déterminer les réaffectations optimales des clients associés. Pour optimiser sa politique de distribution, l'entreprise se pose comme objectif la minimisation à la fois de son coût total de transport et du coût total de transport des clients ${ }^{8}$.

6 Demandes moyennes journalières de l'année 2018.

7 Dans le modèle, une plateforme alimente uniquement des CLRs. Dans cette étude de cas, la plateforme d'Alger pourra par contre alimenter la plateforme de Bouira et certains de ses CLRs dans l'objectif de gérer les pénuries. Pour ce scénario, nous rajoutons la contrainte permettant ces affectations.

${ }^{8}$ Pour le cas spécifique du réseau de Cevital, les termes que nous considérons dans la fonction objectif (d'après les objectifs du groupe) sont : le coût de transport de l'entreprise et des clients et le coût de stockage dans les CLRs. 


\subsection{Analyse et discussion des solutions obtenues}

Après la résolution des modèles associés aux quatre scénarios, nous obtenons les coûts optimaux, que nous résumons dans le Tableau 5.

Tableau 5 - Coûts optimaux de transport (DA/Jour) pour les quatre scénarios.

\begin{tabular}{|c|c|c|}
\hline $\begin{array}{c}\text { Coûts } \\
\text { totaux }\end{array}$ & $\begin{array}{c}\text { Coûts associés à } \\
\text { l'entreprise } \\
\text { (DA) }\end{array}$ & $\begin{array}{c}\text { Coûts associés } \\
\text { aux clients } \\
\text { (DA) }\end{array}$ \\
\hline Scénario 1 & 857142 & 5213531 \\
\hline Scénario 2 & 896302 & 5000768 \\
\hline Scénario 3 & 954508 & 4078603 \\
\hline Scénario 4 & 993668 & 3865840 \\
\hline
\end{tabular}

\section{Scénario 1}

Le coût de transport optimal de l'entreprise (depuis les unités de production jusqu'aux CLRs) est égal à 857142 DA. La somme des coûts de transport des clients (depuis les CLRs jusqu'à leurs sites) est égale à 5213531 DA. Ces deux valeurs représentent les coûts optimaux de transport du réseau actuel de Cevital. Elles nous serviront comme coûts de référence pour comparer avec les résultats des autres scénarios.

\section{Scénario 2}

Le scénario 2 correspond au cas de l'ajout d'une plateforme à Alger qui aura à alimenter la plateforme de Bouira et certains de ses CLRs.

Par ailleurs, les affectations optimales sont comme suit : quelques CLRs seront alimentés à partir de la plateforme d'Alger et d'autres à partir de la plateforme de Bouira. Ces affectations sont résumées dans le Tableau 6.

Tableau 6 - Réaffectation des CLRs pour le cas d'ajout de la plateforme d'Alger.

\begin{tabular}{|c|c|c|}
\hline Plateforme & $\begin{array}{c}\text { Plateforme } \\
\text { d'Alger }\end{array}$ & $\begin{array}{c}\text { Plateforme de } \\
\text { Bouira }\end{array}$ \\
\hline \multirow{2}{*}{ CLR } & $\begin{array}{c}\text { Blida (09), Alger } \\
\text { (16), Boumerdès } \\
(35)\end{array}$ & $\begin{array}{c}\text { Tizi-ouzou } \\
\text { (15), Médéa } \\
(26)\end{array}$ \\
\hline
\end{tabular}

Comparativement au scénario 1 , nous constatons qu'avec l'ajout de la plateforme d'Alger, le coût de transport de l'entreprise augmente (de 39160 DA), alors que celui des clients baisse (de 212763 DA). En effet, ajouter la plateforme d'Alger permettrait d'alimenter certains des CLRs de la plateforme de Bouira et de réduire leurs pénuries de stock. Ainsi, chaque client pourrait s'alimenter auprès d'un seul CLR au lieu de plusieurs, évitant des coûts de transport supplémentaires.

\section{Scénario 3}

Dans le cas où l'on ajoute une plateforme à Biskra, les réaffectations optimales des clients sont présentées dans le Tableau 7.

Tableau 7 - Réaffectation optimale des clients horsCLRs à la plateforme de Biskra.

\begin{tabular}{|c|c|c|c|c|}
\hline \multirow{2}{\Xi}{} & $\begin{array}{c}\text { Biskra } \\
(07)\end{array}$ & $\begin{array}{c}\text { Boussaâda } \\
(28)\end{array}$ & $\begin{array}{c}\text { EL-Oued } \\
(39)\end{array}$ & $\begin{array}{c}\text { Ouargla } \\
(30)\end{array}$ \\
\cline { 2 - 5 } & $\begin{array}{c}\text { Djelfa } \\
(17)\end{array}$ & $\begin{array}{c}\text { Saida } \\
(20)\end{array}$ & $\begin{array}{c}\text { Laghouat } \\
(03)\end{array}$ & $\begin{array}{c}\text { Tamanrasset } \\
(11)\end{array}$ \\
\hline
\end{tabular}

En comparaison avec le scénario 1 de référence, l'ajout de la plateforme de Biskra, située au sud de l'Algérie, permet de tirer les enseignements suivants :

- d'une part, le coût de transport de l'entreprise augmente, car elle serait amenée à acheminer la marchandise des unités de production du Nord jusqu'à la plateforme de Biskra ;

- d'autre part, le coût total de transport des clients diminue. Ce résultat est expliqué par le fait que les huit clients du Sud réaffectés à la plate-forme de Biskra (Cf. Tableau 7) ne seront plus contraints d'aller jusqu'au Nord pour s'alimenter.

\section{Scénario 4}

Dans ce dernier scénario, il s'agit d'étudier le développement du réseau de distribution tel qu'il est planifié par Cevital, consistant en l'ajout d'une plateforme à Alger et une autre à Biskra. Les réaffectations optimales des CLRs et des clients sur les deux nouvelles plateformes sont représentées dans les Tableaux 6 et 7 . 
La lecture des résultats nous permet de conclure que l'ajout de la plateforme d'Alger et de la plateforme de Biskra permet à la fois :

i) une augmentation considérable du coût de transport de l'entreprise par rapport aux scénarios 2 et 3 , expliquée par le fait que les deux plateformes ajoutées doivent être approvisionnées par l'entreprise ellemême à partir des unités de production ;

ii) une réduction considérable du coût de transport des clients. Ce résultat s'explique en grande partie, d'une part par le rôle joué par la plateforme d'Alger dans la couverture d'une agglomération ayant une forte demande et, d'autre part, par le fait d'éviter aux clients du Sud d'effectuer de très longues distances pour s'approvisionner auprès des unités de production du Nord (et donc de leur permettre une économie conséquente).

\section{Délocalisation des CLRs (scénarios 1, 2, 3 et 4)}

A l'issue de notre analyse des quatre scénarios, les résultats suggèrent de supprimer 3 CLRs et recommandent les réaffectations des clients comme suit :
- supprimer le CLR de Bejaia et réaffecter ses clients aux 3 unités de production;

- supprimer le CLR de Constantine et réaffecter ses clients au CLR de Oum-ElBouaghi ;

- supprimer le CLR de Tlemcen et réaffecter ses clients au CLR de Mascara.

Afin de synthétiser et comparer les solutions associées aux quatre scénarios, nous schématisons les résultats du Tableau 5 dans la Figure 4.

A partir de la comparaison des solutions des quatre scénarios, nous pouvons tirer les conclusions principales suivantes :

- l'ajout de la plateforme d'Alger (scénario

2) et de la plateforme de Biskra (scénario

3) engendre pour l'entreprise des coûts de transport supplémentaires, mais permet de réduire les coûts de transport des clients ;

- l'ajout en même temps de la plateforme d'Alger et de la plateforme de Biskra (scénario 4) engendre certes pour l'entreprise des coûts de transport beaucoup plus importants que ceux des scénarios 2 et 3 mais, en contrepartie, permet de réduire énormément les coûts de transport des clients.

Figure 4 - Coûts de transport de l'entreprise Cevital et de ses clients.

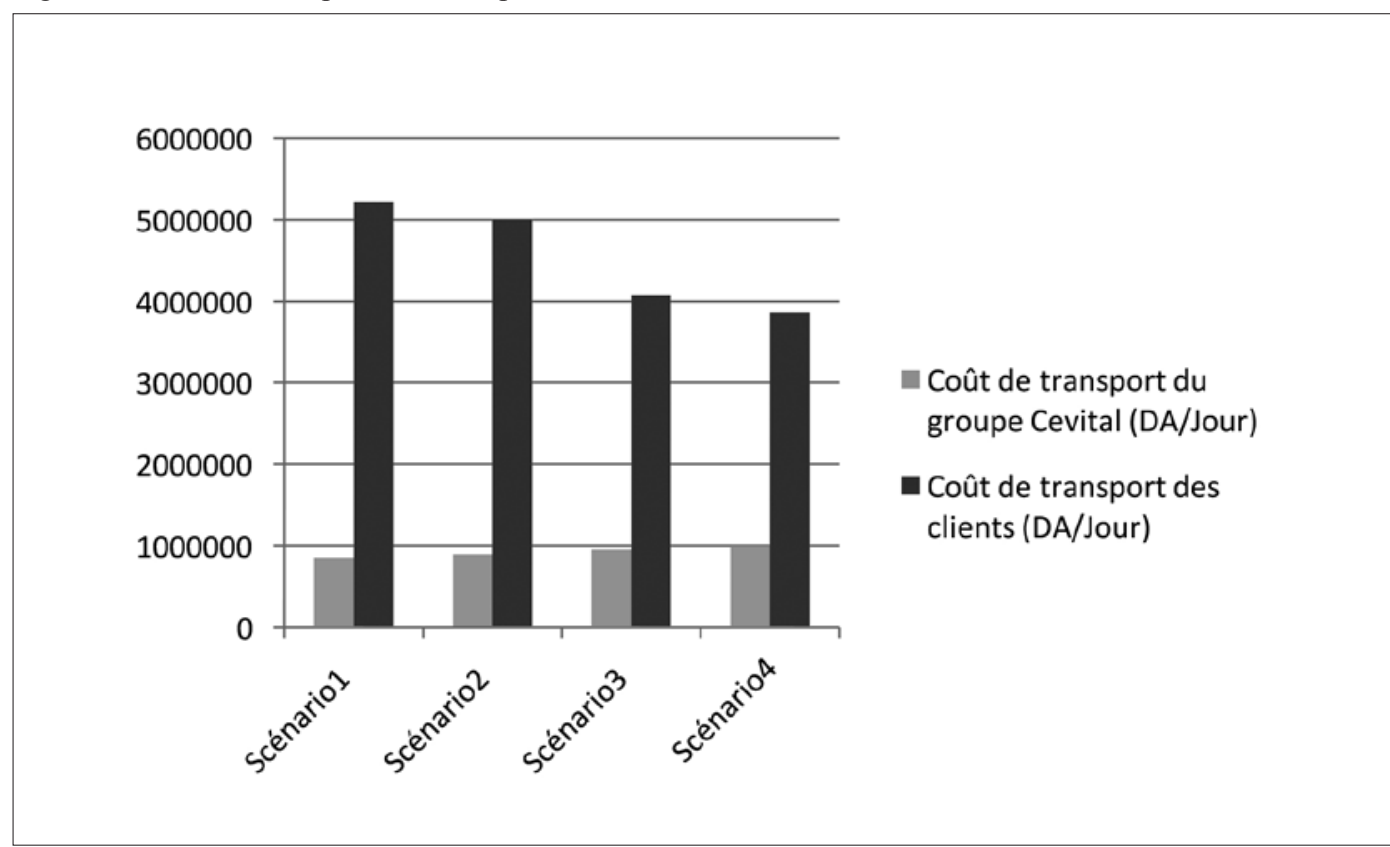


Ainsi, Cevital pourra choisir le plan de distribution qui lui conviendra le plus, tenant compte de son coût de transport, de ses objectifs financiers, des objectifs de rapprochement des produits aux clients et de leur satisfaction.

\section{Conclusion}

Le développement du commerce agricole et agroalimentaire méditerranéen est de plus en plus conditionné par les capacités des pays à se doter d'infrastructures modernes pour faciliter la circulation des marchandises, maximiser la valeur créée par les filières et garantir la qualité des produits offerts (Meziani et al., 2016 ; Driouech et al., 2014). La position géographique stratégique de la région méditerranéenne, reliant trois continents par des routes commerciales et présentant des marchés en croissance potentielle, en fait l'une des zones économiques les plus importantes pour le commerce dans le monde. Cependant, et malgré ce potentiel, la région éprouve des difficultés à jouer ce rôle clé sur le marché mondial. Les faiblesses de la région se concentrent principalement au niveau des pays du Sud et de l'Est de la Méditerranée (PSEM). Parmi les facteurs qui pèsent lourdement sur la performance commerciale des PSEM, les insuffisances logistiques (infrastructures de transport, de conditionnement, de stockage...) apparaissent comme prégnants et constituent l'une des préoccupations majeures des décideurs ${ }^{9}$. Deux études de la Banque mondiale, menées en 2012 et en 2014, pointent clairement du doigt ces insuffisances, notamment pour les PSEM. S'appuyant sur un indice de performance logistique, l'étude de 2014 montre qu'aucun des pays méditerranéens ne figure dans les dix premières positions du classement établi et la majorité ont vu leur po- sition dégradée par rapport au précédent classement de 2012 (voir Banque mondiale, 2014).

Malgré ces insuffisances logistiques, on peut déplorer que les recherches dans ce domaine soient au final peu nombreuses au regard des enjeux associés. Par ailleurs, les études existantes se concentrent le plus souvent, sur le rôle des infrastructures de type publique : infrastructures de stockage (silos, entrepôts frigorifiques en zones de fret...), de transport (routes, transport ferroviaire, aérien...). Il n'existe pas, à notre connaissance, d'analyses portant sur le rôle des réseaux logistiques de type privé (stockage, entreposage, centre de distribution...). Les insuffisances d'organisation des secteurs de la collecte, de l'entreposage, de distribution et de transport des produits agro-alimentaires (absence de services publics, d'entreprises spécialisées, prédominance de l'informel) poussent certains groupes industriels des pays du Sud de la méditerranéen à internaliser les opérations de distribution de leurs produits ou à créer leurs propres filiales de logistique, de distribution ou de transport ${ }^{10}$. Les modèles d'optimisation des réseaux, à l'exemple du modèle que nous proposons, permettent de répondre à un niveau microéconomique, au besoin de ces entreprises en matière de dimensionnement de leur réseau et d'optimisation de son fonctionnement. En s'appuyant sur les données réelles de l'entreprise et sur la structure de son réseau, l'étude a contribué à proposer des solutions opérationnelles, après avoir construit un modèle adapté à la réalité de l'entreprise. De ce point de vue, notre étude comble un manque flagrant de la théorie de la localisation en études de cas, les travaux étant généralement articulés autour de scénarios théoriques donnant lieu à des modélisations originales et des techniques de résolution innovantes (voir ReVelle et Eiselt, 2005) ${ }^{11}$.

\footnotetext{
9 A titre d'exemple, Carruthers (2012) montre qu'une augmentation de $10 \%$ des frais de transport peut réduire de $20 \%$ le volume des échanges et générer une inflation aux effets négatifs sur la compétitivité des économies.

${ }^{10}$ En Algérie par exemple avec le groupe Cevital et sa filiale Numilog, au Maroc avec le groupe Unimer SA et sa filiale de logistique et de distribution VCR Logistics et en Tunisie avec le groupe Poulina, où chacune de ses filiales intègre son propre réseau de distribution.

${ }^{11}$ Comme exceptions, on peut citer par exemple Y1ldiz et al. (2019) qui s'intéressent aux localisations de magasins d'alimentation en Turquie, Wang et al. (2017), pour la localisation de centres de distribution réfrigérés pour une grande chaîne de supermarchés d'une compagnie chinoise.
} 
Si cette approche opérationnelle est nécessaire pour aider les entreprises à concevoir et gérer de façon optimale leurs réseaux propres, l'analyse des performances qui en découlent sur le développement des pays de la région méditerranéenne est, comme souligné précédemment, une question de recherche ouverte. Quel rôle joue de tels réseaux dans les performances économiques de ces pays ? Quelles en sont les externalités sur les circuits de commercialisation, les prix, les capacités de production et de distribution du pays? On peut raisonnablement penser qu'au-delà des retombées directes sur les revenus des entreprises en question, une bonne gestion de ces réseaux peut générer des effets positifs et des effets d'entraînement sur les différents maillons et activités du secteur agro-alimentaire d'un pays. Ces effets pourraient être d'autant plus intéressants à évaluer qu'un certain nombre d'infrastructures privées ont été justement conçues pour pallier les insuffisances et les défaillances des infrastructures publiques. L'évaluation de tels effets constitue de notre point de vue, un enjeu important et un axe de recherche qui mérite d'être davantage développé.

\section{Références}

Ahmadi-Javid A., Hoseinpour P., 2015. A location-inventory-pricing model in a supply chain distribution network with price-sensitive demands and inventory-capacity constraints. Transportation Research, Part E, 82: 238-255.

ANPCE, 2019. Cevital -success stories-. Rapport du Ministère du Commerce, Agence Nationale de la Promotion du Commerce Extérieur. Available at: http:/www.algex.dz/index.php/blog-export/ item/7-Cevital.

Arvis J.F., Saslavsky D., Ojala L., Shepherd B., Busch C., Raj A., 2014. Connecting to compete: trade logistics in the global economy-the logistics performance index and its indicators. Washington, DC: World Bank.

Balinski M.L., 1965. Integer Programming: Methods, Uses, Computations. Management Science, 12: 253-313.

Baumol W.J., Wolfe P., 1976. A Warehouse-Location Problem. In: Mathematical Models in Marketing. Lecture Notes in Economics and Mathematical Systems (Operations Research), vol. 132. Berlin, Heidelberg: Springer.
Bennacer L., 2018. Les industries agroalimentaires : cas de la wilaya de Guelma dans le Nord-Est Algérien. Thèse de Doctorat en Sciences, Département d'Aménagement du territoire, Université des Frères Mentouri-Constantine 1.

Bessaoud O., Pellissier J.P., Rolland J.P., Khechimi W., 2019. Rapport de synthèse sur l'agriculture en Algérie. [Rapport de recherche] CIHEAM-IAMM, pp. 82. hal-02137632.

Biuki M., Kazemi A., Alinezhad A., 2020. An integrated location-routing-inventory model for sustainable design of a perishable products supply chain network. Journal of Cleaner Production, 260: 120842.

Carruthers R., 2012. Transport infrastructure for MED11 countries. CASE Network Reports No. 108.

Cortinhal M.J., Lopes M.J., Melo M.T., 2015. Dynamic design and re-design of multi-echelon, multi-product logistics networks with outsourcing opportunities: A computational study. Computers \& Industrial Engineering, 90: 118-131.

Driouech N., Hmid A., El Bilali H., Lipinska I., Berjan S., 2014. Agri-food logistics in the Mediterranean region: challenges and opportunities. In: K. Wajszczuk K., P. Sajna P., J. Wawrzynowicz J. (eds.), Proceedings of the International Forum on AgriFood Logistics, II Domestic Scientific Conference AGROLOGISTYKA (Poznan, Poland, September 9-13), pp. 48-53.

Etemadnia H., Goetz S.J., Canning P., Tavallali M.S., 2015. Optimal wholesale facilities location within the fruit and vegetables supply chain with bimodal transportation options: An LP-MIP heuristic approach. European Journal of Operational Research, 244: 648-661.

Francis R.L., Mc Ginnis L.F., White J.A., 1983. Locational analysis. European Journal of Operationnal Research, 12(3): 220-252.

Fujita M., Thisse J.F., 1997. Economie géographique, problèmes anciens et nouvelles perspectives. Annales d'Economie et de Statistique, 45: 37-87.

Galati A., Schimmenti E., Carapezza R., 2008. Sicilian floriculture companies and the role of transport in increasing their competitiveness. New Medit, 7(4): 20-28.

Geoffrion A.M., Grave G.W., 1974. Multicommodity Distribution System Design by Benders Decomposition. Management Science, 20(5): 822-844.

Hotelling H., 1929. Stability in competition. Economic Journal, 39: 41-57.

Klose A., Drexl A., 2005. Facility location models for distribution system design. European Journal of Operational Research, 162(1): 4-29. 
Krugman P., 1998. What's new about the new economic geography? Oxford Review of Economic Policy, 14(2): 7-17.

Ma Y., Yan F., Kang K., Wei X., 2016. A novel integrated production-distribution planning model with conflict and coordination in a supply chain network. Knowledge-Based Systems, 105: 119-133.

Melo M.T., Nickel S., Saldanha-Da-Gama F., 2009. Facility location and supply chain management-A review, European Journal of Operational Research, 196(2): 401-412.

Meziani L., Hammoudi A., Radjef M.S., 2016. Mise à niveau logistique, concurrence et sécurité des aliments en Méditerranée: approche théorique et enseignements économiques. New Medit, 15(4): 42-52.

Msaddak M., Ben-Nasr J., Zaibet L., 2019. Resolving recurrent imperfections in the dairy production using gaming simulation, New Medit, 18(4): 35-50.

Padilla M., Bencharif A., 2001. Approvisionnement alimentaire des villes : concepts et méthodes d'analyse des filières et marchés. In: Padilla M., Ben Saïd T., Hassainya J., Le Grusse P. (eds.), Les filières et marchés du lait et dérivés en Méditerranée : état des lieux, problématique et méthodologie pour la recherche. Montpellier: CIHEAM, pp. 262-279 (Options Méditerranéennes : Série B. Etudes et Recherches; n. 32).

Ponsard C., Beguin H., 1988. Analyse économique spatiale. Paris: Presses Universitaires de France.

ReVelle C.S., Eiselt H.A., 2005. Location analysis: A synthesis and survey. European Journal of Operational Research, 165: 1-19.
Rhim H., Ho T.H., Karmarkar U.S., 2003. Competitive location, production, and market selection. European Journal of Operational Research, 149(1): 211-228.

Sarkar J., Gupta B., Pal D., 1997. Location equilibrium for Cournot oligopoly in spatially separated markets. Journal of Regional Science, 37(2): 195-212.

Tang X., Lehuédé F., Péton O., 2016. Location of distribution centers in a multi-period collaborative distribution network. Electronic Notes in Discrete Mathematics, 52: 293-300.

Wang M., Gündüz H.I., Herty M., Zhao L., 2017. Quantity and location decision of fresh food distribution centers for a supermarket chain under carbon policies. In: Proceedings of the 50th Hawaii International Conference on System Sciences, pp. 1361-1370.

Weber A., 1909. Ueber den Standort der industrien. Tübingen: Verlag von J.C.B. Mohr (Paul Siebeck). English translation by Friedrich, C.J., 1929, Theory of the Location of Industries. Chicago: University of Chicago Press.

Yıldız N., Tüysüz F., 2019. A hybrid multi-criteria decision making approach for strategic retail location investment: Application to Turkish food retailing. Socio-Economic Planning Sciences, 68: 100619.

Zheng X., Yin M., Zhang Y., 2019. Integrated optimization of location, inventory and routing in supply chain network design. Transportation Research Part B: Methodological, 121: 1-20. 


\section{Annexe 1}

Tableau 4 - Les demandes des clients-CLRs.

\begin{tabular}{|c|c|c|c|c|c|}
\hline \multirow{2}{*}{ Clients-CLR } & \multicolumn{5}{|c|}{ Demandes (palettes/jour) } \\
\hline & Huile & Sucre & Margarine & $L L K$ & Cojek \\
\hline Oum El Bouaghi (04) & 60 & 50 & 35 & 40 & 15 \\
\hline Batna (05) & 90 & 60 & 30 & 25 & 15 \\
\hline Bejaia (06) & 15 & 10 & 5 & 5 & 5 \\
\hline Blida (09) & 70 & 60 & 30 & 25 & 15 \\
\hline Tlemcen (13) & 40 & 35 & 25 & 15 & 10 \\
\hline Tlemcen (13) & 35 & 30 & 5 & 10 & 5 \\
\hline Tiaret (14) & 50 & 50 & 20 & 15 & 10 \\
\hline Tizi-Ouzou (15) & 70 & 30 & 40 & 10 & 12 \\
\hline Tizi-Ouzou (15) & 40 & 70 & 30 & 20 & 18 \\
\hline Alger (16) & 70 & 100 & 30 & 10 & 5 \\
\hline Alger (16) & 70 & 40 & 80 & 5 & 0 \\
\hline Alger (16) & 110 & 60 & 40 & 15 & 15 \\
\hline Setif (19) & 50 & 35 & 25 & 10 & 12 \\
\hline Setif (19) & 20 & 35 & 45 & 10 & 8 \\
\hline Sidi Bel Abbès(22) & 35 & 30 & 15 & 10 & 10 \\
\hline Annaba (23) & 65 & 50 & 35 & 20 & 10 \\
\hline Constantine (25) & 25 & 20 & 15 & 5 & 5 \\
\hline Médéa (26) & 40 & 35 & 15 & 10 & 10 \\
\hline Mostaganem (27) & 60 & 50 & 35 & 20 & 10 \\
\hline Mascara (29) & 45 & 30 & 20 & 15 & 15 \\
\hline Oran (31) & 120 & 110 & 30 & 5 & 5 \\
\hline Oran (31) & 20 & 80 & 10 & 5 & 15 \\
\hline Oran (31) & 60 & 70 & 10 & 0 & 5 \\
\hline Boumerdès (35) & 40 & 30 & 45 & 20 & 5 \\
\hline Boumerdès (35) & 20 & 40 & 25 & 10 & 15 \\
\hline Relizane (48) & 60 & 55 & 45 & 30 & 20 \\
\hline
\end{tabular}




\section{Annexe 2}

\section{Description du Modèle (1)}

\section{Les données du problème}

Pour définir les entités de la chaine de distribution agroalimentaire, nous disposons de :

- I: un ensemble d'unités de production ;

- $\mathrm{P}_{\mathrm{e}}$ (resp. $\mathrm{L}_{\mathrm{e}}$ : un ensemble de plateformes (resp. de centres de livraison régionaux (CLRs)) en activités ;

- $\mathrm{P}_{\mathrm{n}}\left(\right.$ resp. $\left.\mathrm{L}_{\mathrm{n}}\right)$ : un ensemble de sites candidats pour la localisation de nouvelles plateformes (resp. de nouveaux CLRs) ;

- $\mathrm{J}$ : un ensemble de clients ;

- $\mathrm{P}=\mathrm{P}_{\mathrm{e}} \cup \mathrm{P}_{\mathrm{n}}:$ l'ensemble des plateformes ;

- $\mathrm{L}=\mathrm{L}_{\mathrm{e}} \cup \mathrm{L}_{\mathrm{n}}$ : l'ensemble des CLRs ;

- M: l'ensemble des produits impliqués dans la chaine de distribution.

Par ailleurs, nous notons les données de la chaine utilisées dans le modèle comme suit :

- $c^{1}$ : le coût unitaire de transport des produits assumé par l'entreprise ;

- $c^{2}$ : le coût unitaire de transport des produits assumé par les clients ;

- $c_{p}^{3}$ : le coût de stockage dans la plateforme en activité, $p, p \in P_{e}$;

- $c_{l}^{4}$ : le coût de stockage dans le CLR en activité, $l, l \in l_{e}$;

- $c_{p}^{5}$ : le coût de fermeture de la plateforme en activité $p, p \in P_{e}$;

- $c_{l}^{6}$ : le coût de fermeture du CLR en activité $l, l \in l_{e}$;

- $c^{7}\left(\right.$ resp. $\left.c^{8}\right)$ : le coût unitaire de construction d'une nouvelle plateforme (resp. d'un nouveau CLR);

- $c^{9}$ (resp. $\left.c^{10}\right)$ : le coût fixe de construction d'une nouvelle plateforme (resp. d'un nouveau CLR);

- $O_{i}^{m}$ : quantité produite du produit par l'unité de production, $i, i \in I, m \in M$;

- $D_{j}^{m}$ : quantité demandée du clientpour le produit $m, j \in J, m \in M$;

- $S_{p}^{1}$ (resp. $\left.R_{p}^{1}\right)$ : capacité maximale (resp. minimale) de la plateforme $p, p \in P$

- $S_{l}^{2}$ (resp. $R_{l}^{2}$ ): capacité maximale (resp. minimale) du CLR $l, l \in L$;

- $d_{i p}^{1}$ : distance entre l'unité de production $i$ et la plateforme $p, i \in I, p \in P$;

- $d_{p l}^{2}$ : distance entre la plateforme $p$ et le $\operatorname{CLR} l, p \in P, l \in L$;

- $d_{l j}^{3}$ : distance entre le CLR $l$ et le client $j, l \in L, j \in J$.

\section{Les variables de décision}

Les variables de décision du modèle sont :

- $x_{i p}^{m}$ : quantité du produit $m$ à transporter de l'unité de production $i$ à la plateforme $p, i \in I, p \in P$, $m \in M$;

- $y_{p l}^{m}$ : quantité du produit $m$ à transporter de la plateforme $p$ au CLR $l, p \in P, l \in L, m \in M$;

- $z_{l j}^{m}$ : quantité du produit $m$ à transporter du CLR $l$ au client $j, m \in M, l \in L, j \in J$;

- $u_{p}^{e}$ : variable booléenne égale à 1 si une plateforme en activité $p$ est à fermer, $p \in P_{e}$;

- $v_{l}^{e}$ : variable booléenne égale à 1 si un CLR en activité $l$ est à fermer, $l \in L_{e}$;

- $u_{p}^{n}$ : variable booléenne égale à 1 si la nouvelle plateforme $p$ est retenue pour être construite, $p$ $\in P_{n}$

- $v_{l}^{n}$ : variable booléenne égale à 1 si un nouveau CLR $l$ est retenu pour être construit, $l \in L_{n}$;

- $\mathrm{x}=\left(\left(x_{i p}^{m}, p \in P, l \in L, m \in M\right),\left(y_{p l}^{m}, p \in P, l \in L, m \in M\right),\left(z_{l j}^{m}, m \in M, l \in L, j \in J\right),\left(u_{p}^{e}, p \in P_{e}\right)\right.$ $\left.\left(v_{l}^{e}, l \in L_{e}\right),\left(u_{p}^{n}, p \in P_{n}\right),\left(v_{l}^{n}, l \in L_{n}\right)\right)$ - le vecteur des décisions. 


\section{La fonction économique (objectif)}

$$
\begin{aligned}
\varphi(x)=c^{1}( & \left.\sum_{i \in I} \sum_{p \in P} d_{i p}^{1} \sum_{m \in M} x_{i p}^{m}+\sum_{p \in P} \sum_{l \in L} d_{p l}^{2} \sum_{m \in M} y_{p l}^{m}\right)+c^{2}\left(\sum_{l \in L} \sum_{j \in J} d_{l j}^{3} \sum_{m \in M} z_{l j}^{m}\right) \\
& +\sum_{p \in P_{e}} c_{p}^{3}\left(1-u_{p}^{e}\right)+\sum_{l \in L_{e}} c_{l}^{4}\left(1-v_{l}^{e}\right)+\sum_{p \in P_{e}} c_{p}^{5} u_{p}^{e}+\sum_{l \in L_{e}} c_{l}^{6} v_{l}^{e} \\
& +\sum_{p \in P_{n}}\left(c^{7} u_{p}^{n}+c^{9} \sum_{i \in I} \sum_{m \in M} x_{i p}^{m}\right)+\sum_{l \in L_{n}}\left(c^{8} v_{l}^{n}+c^{10} \sum_{p \in P_{e}} \sum_{m \in M} y_{p l}^{m}\right)
\end{aligned}
$$

\section{Contraintes}

$\sum_{p \in P} x_{i p}^{m} \leq O_{i}^{m}, \quad \forall i \in I, \quad \forall m \in M ;$

$\sum_{l \in L} z_{l j}^{m}=\mathrm{D}_{\mathrm{j}}^{\mathrm{m}}, \quad \forall j \in J, \quad \forall m \in M ;$

$\sum_{i \in \boldsymbol{I}} \boldsymbol{x}_{\boldsymbol{i} \boldsymbol{m}}^{m}=\sum_{l \in \boldsymbol{L}} \boldsymbol{y}_{p l}^{m}, \quad \forall p \in P, \quad \forall m \in M ;$

$\sum_{p \in P} y_{p l}^{m}=\sum_{j \in J} z_{l j}^{m}, \quad \forall l \in L, \quad \forall m \in M ;$

$\sum_{i \in I} \sum_{m \in M} \boldsymbol{x}_{i p}^{m} \leq\left(1-\boldsymbol{u}_{p}^{e}\right) \boldsymbol{S}_{p}^{1}, \quad \forall p \in P^{e} ;$

$\sum_{i \in I} \sum_{m \in M} \boldsymbol{x}_{i p}^{m} \leq \boldsymbol{n}_{p}^{n} \boldsymbol{S}_{p}^{1}, \quad \forall p \in P^{n}$

$\sum_{i \in I} \sum_{m \in M} x_{i p}^{m} \geq\left(1-u_{p}^{e}\right) R_{p}^{1}, \quad \forall p \in P^{e} ;$

$\sum_{i \in I} \sum_{m \in M} x_{i p}^{m} \geq \boldsymbol{n}_{p}^{n} \boldsymbol{R}_{p}^{1}, \quad \forall p \in P^{n} ;$

$\sum_{p \in P} \sum_{m \in M} y_{p l}^{m} \leq\left(1-v_{p}^{e}\right) S_{l}^{2} \quad \forall l \in L^{e} ;$

$\sum_{p \in P} \sum_{m \in M} y_{p l}^{m} \leq v_{p}^{n} S_{l}^{2}, \quad \forall l \in L^{n} ;$

$\sum_{p \in P} \sum_{m \in M} y_{p l}^{m} \geq\left(1-v_{p}^{e}\right) R_{l}^{2}, \quad \forall l \in L^{e} ;$

$\sum_{p \in P} \sum_{m \in M} y_{p l}^{m} \geq \boldsymbol{v}_{p}^{n} \boldsymbol{R}_{l}^{2}, \quad \forall l \in L^{n} ;$

$\boldsymbol{x}_{\boldsymbol{i} p}^{\boldsymbol{m}} \geq \mathbf{0}, \quad \forall i \in I, \quad \forall p \in P, \quad \forall m \in M ;$

$\boldsymbol{y}_{\boldsymbol{p l}}^{\boldsymbol{m}} \geq \mathbf{0}, \quad \forall p \in P, \quad \forall l \in L, \quad \forall m \in M ;$

$z_{l j}^{m} \geq \mathbf{0}, \quad \forall l \in L, \quad \forall j \in J, \quad \forall m \in M ;$

$\boldsymbol{u}_{\boldsymbol{p}}^{\boldsymbol{e}}, \boldsymbol{u}_{\boldsymbol{p}}^{\boldsymbol{n}} \in\{0,1\}, \quad \forall p \in P ;$

$v_{l}^{e}, v_{l}^{n} \in\{0,1\}, \quad \forall l \in L$. 
L'ensemble $X$ des solutions réalisables du modèle est constitué des vecteurs de décisions vérifiant les contraintes $(A 2.1)-(A 2.18)$.

Le modèle mathématique obtenu est un programme linéaire mixte ${ }^{12}$ (MILP). La fonction objective du modèle, donnée dans $(A 2.1)$, référence le coût total du réseau. Les coûts logistiques (transport de marchandise) sont donnés dans les trois premiers termes : le coût de transport entre les unités de production et les plateformes dans le premier terme ; le coût de transport entre les plateformes et les CLRs dans le deuxième terme ; et celui entre les CLRs et les clients dans le troisième. Les coûts stratégiques (installation et fermeture des centres) sont donnés dans les termes restants : le coût de maintien des plateformes (resp. des CLRs) en activité dans le quatrième (resp. le cinquième) terme ; le coût de fermeture des plateformes (resp. des CLRs) en activité dans le sixième (resp. le septième) terme ; le coût de construction (fixe et variable) de nouvelles plateformes (resp. de nouveaux CLRs) dans le huitième (resp. le neuvième) terme.

Les contraintes (A2.2) assurent que chaque unité de production ne peut délivrer plus qu'elle en possède pour chaque produit. Les contraintes $(A 2.3)$ garantissent que la demande de chaque client pour chaque produit soit satisfaite. Les contraintes $(A 2.4)$ assurent que la marchandise reçue par chaque plateforme en provenance de toutes les unités de production devrait être transférée aux CLRs. Les équations (A2.5) assurent que la marchandise reçue par chaque CLR en provenance des plateformes devrait être transférée aux clients. Les contraintes (A2.6) (resp. (A2.7)) représentent la limitation en capacité des plateformes en activité (resp. à localiser). Les contraintes $(A 2.8)$ (resp. (A2.9)) fixent les capacités minimales que doivent avoir les plateformes en activité (resp. à localiser). Les contraintes (A2.10) (resp. (A2.11)) représentent la limitation en capacité des CLRs en activité (resp. à localiser). Les contraintes (A2.12) (resp. (A2.13)) fixent les capacités minimales que doivent avoir les CLRs en activité (resp. à localiser). Les contraintes $(A 2.14)-(A 2.16)($ resp. $(A 2.17)-(A 2.18))$ sont les contraintes de non négativité (resp. d'intégralité).

${ }^{12}$ Le terme mixte est relatif au fait que certaines variables de décision sont réelles et d'autres sont entières binaires. 